\title{
Generalization of the Zlámal condition for simplicial finite elements in $\mathbf{R}^{d}$
}

\author{
Jan Brandts ${ }^{1}$, Sergey Korotov ${ }^{2}$, Michal Křřžek ${ }^{3, *}$ \\ November 9, 2010 \\ ${ }^{1}$ Korteweg-de Vries Institute, University of Amsterdam \\ Science Park 904, 1098 XH Amsterdam, Netherlands \\ e-mail: janbrandts@gmail.com \\ ${ }^{2}$ BCAM - Basque Center for Applied Mathematics \\ Bizkaia Technology Park, Building 500, E-48160 Derio \\ Basque Country, Spain \\ e-mail: korotov@bcamath.org \\ ${ }^{3}$ Institute of Mathematics, Academy of Sciences \\ Žitná 25, CZ-115 67 Prague 1, Czech Republic \\ e-mail: krizek@math.cas.cz
}

\begin{abstract}
The famous Zlámal's minimum angle condition is widely used for construction of a regular family of triangulations (containing nondegenerating triangles) as well as in convergence proofs for the finite element method in $2 d$. In this paper we shall present and discuss its generalization to simplicial partitions in any space dimension.
\end{abstract}

Keywords: linear finite element, mesh regularity, minimum angle condition, convergence, higher-dimensional problems

Mathematics Subject Classification: 65N12, 65N30, 65N50

\section{Introduction}

The finite element method (FEM) is nowadays one of the most powerful and popular numerical techniques widely used in various software packages that solve problems in, for instance, mathematical physics and mechanics. The initial step in FEM implementations is to establish an appropriate partition (also called mesh, grid, triangulation, etc.) of the solution domain. For a number of applications and complicated geometries, simplicial partitions are prefered over the others due to their flexibility. However, from both

* The second author was partially supported by Grant MTM2008-03541 of the MICINN, Spain, the ERC Advanced Grant FP7-246775 NUMERIWAVES and Grant PI2010-04 of the Basque Government. The third author was supported by Grant no. IAA 100190803 of the Grant Agency of the Academy of Sciences of the Czech Republic and the Institutional Research Plan AV0Z 10190503. 
theoretical and practical points of view, such partitions and their refinements cannot be constructed arbitrarily. Thus, first of all we must ensure, at least theoretically, that the finite element approximations converge to the exact (weak) solution of the mathematical model under consideration when the associated partitions become finer [5]. Mainly due to this reason the notions of regular families of partitions or shape-preserving partitions appeared. Second, the regularity is also important for real-life computations, because partitions that contain almost flat (i.e. degenerating) elements may yield ill-conditioned stiffness matrices [10].

In 1968, Miloš Zlámal [12] introduced the so-called minimum angle condition that ensures the convergence of the finite element approximations for solving linear elliptic boundary value problems of the second and fourth order on triangular meshes. This condition requires that there exists a constant $\alpha_{0}>0$ such that the minimal angle $\alpha_{S}$ of each triangle $S$ in all triangulations used satisfies

$$
\alpha_{S} \geq \alpha_{0} .
$$

In fact, Zlámal used the equivalent condition $\sin \alpha_{S} \geq \sin \alpha_{0}$. The same condition was also introduced by Alexander Ženíšek [11] for the finite element method applied to a system of linear elasticity equations of second order published in 1969. However, this paper was submitted already on April 3, 1968, whereas Zlámal's paper on April 17, 1968.

Later, the so-called inscribed ball condition was introduced, see, e.g. [5, p. 124], which uses a ball contained in a given element (cf. (2)). Thus, it can also be used for nonsimplicial elements in any dimension. This condition has an elegant geometrical interpretation: the ratio of the radius of the inscribed ball of any element and the diameter of this element must be bounded from below by a positive constant over all partitions. Roughly speaking, no element of no partitions should degenerate to a hyperplane as the discretization parameter $h$ (which is the maximal diameter of all elements in the corresponding partition) tends to zero. This property is called in [5] the regularity of a family of partitions. For triangular elements it is, obviously, equivalent to Zlámal's condition. This condition has a large number of applications, see e.g. [5, 7, 11].

In [8] the inscribed ball condition (cf. (2)) was replaced by a simpler equivalent condition on the volume of every element (cf. (1)). Another equivalent circumscribed ball condition for simplices (cf. (3)) was first introduced in [4].

In the present paper Zlámal's condition will be generalized into $\mathbf{R}^{d}$ for any $d \in$ $\{2,3, \ldots\}$ so that the $d$-dimensional sinus of all angles at vertices of all simplices (as defined in [6]) is bounded from below by a positive constant. This will be proved to be equivalent with the inscribed ball condition.

\section{On mesh regularity conditions}

A simplex $S$ in $\mathbf{R}^{d}, d \in\{1,2,3, \ldots\}$, is the convex hull of $d+1$ vertices $A_{1}, A_{2}, \ldots, A_{d+1}$ that do not belong to the same $(d-1)$-dimensional hyperplane, i.e., $S=\operatorname{conv}\left\{A_{1}, A_{2}, \ldots, A_{d+1}\right\}$. We denote by $h_{S}$ the length of the longest edge of $S$.

Let

$$
F_{i}=\operatorname{conv}\left\{A_{1}, \ldots, A_{i-1}, A_{i+1}, \ldots, A_{d+1}\right\}
$$

be the facet of $S$ opposite to vertex $A_{i}$ for $i \in\{1, \ldots, d+1\}$. The dihedral angle $\alpha$ between two such facets is defined by means of the inner product of their outward unit normals 
$n_{1}$ and $n_{2}$,

$$
\cos \alpha=-n_{1} \cdot n_{2}
$$

see also $[6$, p. 74$]$.

Let $\Omega \subset \mathbf{R}^{d}$ be a bounded domain. Assume that $\bar{\Omega}$ is polytopic. By this we mean that $\bar{\Omega}$ is the closure of a domain whose boundary $\partial \bar{\Omega}$ is contained in a finite number of $(d-1)$-dimensional hyperplanes.

Next we define a simplicial partition of a bounded closed polytopic domain $\bar{\Omega} \subset \mathbf{R}^{d}$ as follows. We subdivide $\bar{\Omega}$ into a finite number of simplices (called elements and denoted by $S$ ), so that their union is $\bar{\Omega}$, any two distinct simplices have disjoint interiors, and any facet of any simplex is either a facet of another simplex from the partition or belongs to the boundary $\partial \bar{\Omega}$. The set of such simplices will be called simplicial partition and denoted by $\mathcal{T}_{h}$, where $h=\max _{S \in \mathcal{T}_{h}} h_{S}$.

The sequence of partitions $\mathcal{F}=\left\{\mathcal{T}_{h}\right\}_{h \rightarrow 0}$ of $\bar{\Omega}$ is called a family of partitions if for every $\varepsilon>0$ there exists $\mathcal{T}_{h} \in \mathcal{F}$ with $h<\varepsilon$.

In what follows, all constants $C_{i}$ are independent of $S$ and $h$, but can depend on the dimension $d$. By $\operatorname{vol}_{p}$ we denote the $p$-dimensional volume $(p \leq d)$.

The equivalence of the following three regularity conditions for simplicial partitions in $\mathbf{R}^{d}$ was proved in [3] for any $d \geq 2$. They guarantee the optimal order of the interpolation error of simplicial finite elements, which is employed in various convergence proofs of the finite element method [5].

Condition 1: There exists $C_{1}>0$ such that for any $\mathcal{T}_{h} \in \mathcal{F}$ and any $S \in \mathcal{T}_{h}$ we have

$$
\operatorname{vol}_{d} S \geq C_{1} h_{S}^{d}
$$

Condition 2: There exists $C_{2}>0$ such that for any $\mathcal{T}_{h} \in \mathcal{F}$ and any $S \in \mathcal{T}_{h}$ we have

$$
\operatorname{vol}_{d} b \geq C_{2} h_{S}^{d},
$$

where $b \subset S$ is the inscribed ball of $S$.

Condition 3: There exists $C_{3}>0$ such that for any $\mathcal{T}_{h} \in \mathcal{F}$ and any $S \in \mathcal{T}_{h}$ we have

$$
\operatorname{vol}_{d} S \geq C_{3} \operatorname{vol}_{d} B
$$

where $B \supset S$ is the circumscribed ball about $S$.

If one of the above conditions (1)-(3) is satisfied, the family $\mathcal{F}$ of simplicial partitions is called regular. In addition, in [2] the following minimum angle condition was introduced and its equivalence with the above three, (1), (2), and (3), was proved for $d=2,3$ :

Condition 4: There exists a constant $C_{4}>0$ such that for any partition $\mathcal{T}_{h} \in \mathcal{F}$, any simplex $S \in \mathcal{T}_{h}$, and any dihedral angle $\alpha$ and for $d=3$ also any angle $\alpha$ within any triangular face of $S$, we have

$$
\alpha \geq C_{4}
$$

However, the problem how to formulate Condition 4 in dimensions higher than 3 so that it is equivalent to Conditions $\mathbf{1}, \mathbf{2}$, and $\mathbf{3}$ (or just to one of them) remained open. In order to fill this gap, in the next section we shall present a definition of $d$-dimensional sine used by Folke Eriksson in [6]. 


\section{The minimum angle condition in $\mathbf{R}^{d}$}

A convenient definition for the $d$-dimensional sine of angles in $\mathbf{R}^{d}$ was introduced in [6]. In terms of the simplex $S$, for any of its vertices $A_{i}$, the $d$-dimensional sine of the angle of $S$ at $A_{i}$, denoted by $\hat{A}_{i}$, is defined as follows (see (3) in [6, p. 72]):

$$
\sin _{d}\left(\hat{A}_{i} \mid A_{1} A_{2} \ldots A_{d+1}\right)=\frac{d^{d-1}\left(\operatorname{vol}_{d} S\right)^{d-1}}{(d-1) ! \Pi_{j=1, j \neq i}^{d+1} \operatorname{vol}_{d-1} F_{j}} .
$$

Remark 1 For $d=2, \sin _{2}\left(\hat{A}_{i} \mid A_{1} A_{2} A_{3}\right)$ is the standard sine of the angle $\hat{A}_{i}$ in the triangle $A_{1} A_{2} A_{3}$, due to the following well-known formula, e.g. for $i=1$,

$$
\operatorname{vol}_{2}\left(A_{1} A_{2} A_{3}\right)=\frac{1}{2}\left|A_{1} A_{2}\right|\left|A_{1} A_{3}\right| \sin \hat{A}_{1} .
$$

In fact, one can similarly define a sine for any $k$-dimensional (vertex) angle of any $k$-dimensional facet of $S$ for $k \in\{2, \ldots, d\}$. Namely, let us denote the $k$-dimensional facet of $S$ spanned by the $k+1$ (distinct) vertices $A_{i_{1}}, A_{i_{2}}, \ldots, A_{i_{k+1}}$ by $F_{i_{1}, i_{2}, \ldots, i_{k+1}}$. Then for any index $i_{\ell} \in\left\{i_{1}, \ldots, i_{k+1}\right\}$ we set

$$
\sin _{k}\left(\hat{A}_{i_{\ell}} \mid A_{i_{1}} A_{i_{2}} \ldots A_{i_{k+1}}\right)=\frac{k^{k-1}\left(\operatorname{vol}_{k} F_{i_{1}, i_{2}, \ldots, i_{k+1}}\right)^{k-1}}{(k-1) ! \Pi_{i_{j} \in\left\{\left\{i_{1}, \ldots, i_{k+1}\right\} \backslash\left\{i_{\ell}\right\}\right\}} \operatorname{vol}_{k-1} F_{i_{1}, i_{2}, \ldots, i_{k+1}}^{i_{i}}}
$$

where $F_{i_{1}, i_{2}, \ldots, i_{k+1}}^{i_{j}}$ denotes $(k-1)$-dimensional facet of $F_{i_{1}, i_{2}, \ldots, i_{k+1}}$ (which is clearly itself a $(k-1)$-dimensional simplex) lying against the vertex $A_{i_{j}}$.

Remark 2 Notice that in the above denotation we have $S \equiv F_{1,2, \ldots, d+1}$ and $F_{j} \equiv F_{1,2, \ldots, d+1}^{j}$ for $j=1,2, \ldots, d+1$.

The above definition of sine inspires the following:

Generalized Condition 4: There exists $C_{4}^{\prime}>0$ such that for any $\mathcal{T}_{h} \in \mathcal{F}$ and any $S=\operatorname{conv}\left\{A_{1}, \ldots, A_{d+1}\right\} \in \mathcal{T}_{h}$ we have

$$
\sin _{d}\left(\hat{A}_{i} \mid A_{1} A_{2} \ldots A_{d+1}\right) \geq C_{4}^{\prime}>0 \quad \forall i \in\{1,2, \ldots, d+1\},
$$

where $\sin _{d}$ is defined in (5).

From Remark 1 we observe that (8) really presents a generalization of Zlámal's condition to higher dimensions, i.e. for $d \geq 3$.

Remark 3 The angle $\hat{A}_{i}$ at vertex $A_{i}$ defined in (5) is, in general, not equal to the solid angle as it could be guessed. For instance, for the regular tetrahedron the solid angle is $3 \arccos 1 / 3-\pi \approx 0.5513$ steradians (see [9, p. 83]), whereas $\sin _{3}\left(A_{i} \mid A_{1} A_{2} A_{3} A_{4}\right)=4 \sqrt{3} / 9$ and $\arcsin 4 \sqrt{3} / 9 \approx 0.8785$. 


\section{Equivalence of Condition 1 and Generalized Con- dition 4 in any dimension}

First, we present some useful results.

Proposition 1 For any simplex $S\left(\equiv F_{1,2, \ldots, d+1}\right)$ and any its $k$-dimensional facet $F_{i_{1}, i_{2}, \ldots, i_{k+1}}$ we have

$$
\operatorname{vol}_{k} F_{i_{1}, i_{2}, \ldots, i_{k+1}} \leq h_{S}^{k}
$$

P r o o f : Relation (9) follows from the fact that the distance between any two points of $S$ is not larger than $h_{S}$. Thus, $S$ and its facets are contained in hypercubes (of the corresponding dimensions) with edges of length $h_{S}$.

Proposition 2 Let condition (1) hold for the family $\mathcal{F}$. Then there exists $C_{5}>0$ such that for any lower dimensional facet $F_{i_{1}, i_{2}, \ldots, i_{k+1}}$ of any simplex $S$ from any $\mathcal{T}_{h} \in \mathcal{F}$ we have the following lower estimate

$$
\operatorname{vol}_{k} F_{i_{1}, i_{2}, \ldots, i_{k+1}} \geq C_{5} h_{S}^{k} .
$$

P r o o f : First, we demonstrate how to prove (10) for $(d-1)$-dimensional facets $F_{j}$. Write $v_{j}$ for the altitude of the vertex $A_{j}$ to the face $F_{j}$. Then, since $v_{j} \leq h_{S}$, we observe that (i.e. for $k=d-1$ )

$$
C_{1} h_{S}^{d} \leq \operatorname{vol}_{d} S=\frac{1}{d} v_{j} \operatorname{vol}_{d-1} F_{j} \leq \frac{1}{d} h_{S} \operatorname{vol}_{d-1} F_{j},
$$

from which (10) follows immediately. By induction, we can easily prove (10) for the other (lower-dimensional) facets of $S$ in the same manner.

Theorem 1 Conditions (1) and (8) are equivalent in $\mathbf{R}^{d}$ for any $d \geq 2$.

P r o o f : Condition $1 \Longrightarrow$ Generalized Condition 4: Let $i \in\{1, \ldots, d+1\}$ be given. Then from (5), (1), and (9) we immediately observe that

$$
\begin{gathered}
\sin _{d}\left(\hat{A}_{i} \mid A_{1} A_{2} \ldots A_{d+1}\right)=\frac{d^{d-1}\left(\operatorname{vol}_{d} S\right)^{d-1}}{(d-1) ! \Pi_{j=1, j \neq i}^{d+1} \operatorname{vol}_{d-1} F_{j}} \geq \\
\geq \frac{d^{d-1}\left(C_{1} h_{S}^{d}\right)^{d-1}}{(d-1) ! h_{S}^{(d-1) d}}=\frac{d^{d-1}}{(d-1) !} C_{1}^{d-1}=C_{4}^{\prime}>0 .
\end{gathered}
$$

Generalized Condition $4 \Longrightarrow$ Condition 1: By [6, p. 76] and (8), we get for any $i_{\ell} \in\left\{i_{1}, i_{2}, i_{3}\right\}$ that

$$
\begin{gathered}
\sin _{2}\left(\hat{A}_{i_{\ell}} \mid A_{i_{1}} A_{i_{2}} A_{i_{3}}\right) \geq \sin _{3}\left(\hat{A}_{i_{\ell}} \mid A_{i_{1}} A_{i_{2}} A_{i_{3}} A_{i_{4}}\right) \geq \ldots \\
\geq \sin _{d}\left(\hat{A}_{i_{\ell}} \mid A_{1} \ldots A_{d+1}\right) \geq C_{4}^{\prime}
\end{gathered}
$$

where $i_{1}, i_{2}, i_{3}, i_{4}, \ldots$ are distinct indices from the set $\{1,2, \ldots, d+1\}$. From this and $(7)$ we have for $k=2,3, \ldots, d$ the following relation

$$
\left(\operatorname{vol}_{k} F_{i_{1}, i_{2}, \ldots, i_{k+1}}\right)^{k-1}=
$$




$$
\begin{gathered}
=\frac{(k-1) !}{k^{k-1}}\left(\Pi_{i_{j} \in\left\{\left\{i_{1}, \ldots, i_{k+1}\right\} \backslash\left\{i_{\ell}\right\}\right\}} \operatorname{vol}_{k-1} F_{i_{1}, i_{2}, \ldots, i_{k+1}}^{i_{j}}\right) \sin _{k}\left(\hat{A}_{i_{\ell}} \mid A_{i_{1}} A_{i_{2}} \ldots A_{i_{k+1}}\right) \geq \\
\geq \frac{(k-1) !}{k^{k-1}} C_{4}^{\prime} \Pi_{i_{j} \in\left\{\left\{i_{1}, \ldots, i_{k+1}\right\} \backslash\left\{i_{\ell}\right\}\right\}} \operatorname{vol}_{k-1} F_{i_{1}, i_{2}, \ldots, i_{k+1}}^{i_{j}}
\end{gathered}
$$

for any $i_{\ell} \in\left\{i_{1}, \ldots, i_{k+1}\right\}$. Now we apply (12) several times in order to estimate $\operatorname{vol}_{d} S$ from below by a product of some constant which only depends on $k$ and the given constant $C_{4}^{\prime}$ from (8) times lengths of some $d$ edges of $S$.

Further, in order to finally prove (1), it remains to show that there exists a constant $C_{6}>0$ such that for any simplex $S$ from any $\mathcal{T}_{h} \in \mathcal{F}$ and any edge $A_{m} A_{n}$ of $S$ we have $\left|A_{m} A_{n}\right| \geq C_{6} h_{S}$. In order to show that let us take any edge in $S$. Without loss of generality, let it be $A_{1} A_{2}$ and assume that $h_{S}=\left|A_{i} A_{j}\right|$ for some $i$ and $j$. We consider first the triangle $A_{1} A_{2} A_{3}$, where by the law of sines and (11) one has

$$
\left|A_{1} A_{2}\right| \geq\left|A_{1} A_{2}\right| \sin \hat{A}_{1}=\left|A_{2} A_{3}\right| \sin \hat{A}_{3} \geq C_{4}^{\prime}\left|A_{2} A_{3}\right| .
$$

Further, we consider the triangle $A_{2} A_{3} A_{4}$, where we show that $\left|A_{2} A_{3}\right| \geq C_{4}^{\prime}\left|A_{3} A_{4}\right|$, etc. Finally, using all the inequalities obtained, we get that $\left|A_{1} A_{2}\right| \geq C_{6}\left|A_{i} A_{j}\right|=C_{6} h_{S}$, where $C_{6}$ depends only on the dimension $d$ and the given constant $C_{4}^{\prime}$.

Corollary 1 Conditions 1, 2, 3, and Generalized Condition 4 are equivalent for any $d \geq 2$.

Remark 4 Condition (4) is clearly equivalent to Generalized Condition 4 when $d \in$ $\{2,3\}$, since both are equivalent, e.g., to the inscribed ball condition (2). In [6, Sect. 5], one can find useful relations between, e.g., 3-dimensional sine and dihedral angles and angles between edges of a tetrahedron.

\section{Final remarks}

As an eye visualization is almost impossible in dimensions higher than three, the concept of "measuring" angles due to F. Eriksson is found to be very convenient (uniform and compact) for any dimension.

Moreover, the original estimates of Zlámal [12, p. 397] from which his condition is derived are, in fact, using the sine of two-dimensional angles themselves.

Zlámal's minimum angle can be generalized to the maximum angle condition (mostly for $d=2,3$ ) which represents a weaker sufficient condition guaranteeing the convergence of the finite element method. The maximum angle condition enables us to treat anisotropic partitions (see e.g. [1]) containing flat elements. They can yield ill-conditioned stiffness matrices, but the optimal interpolation order may be preserved.

\section{References}

[1] Apel, T., Anisotropic Finite Elements: Local Estimates and Applications, Advances in Numerical Mathematics. B. G. Teubner, Stuttgart, 1999. Zbl 0934.65121

[2] Brandts, J., Korotov, S., KřížEK, M., On the equivalence of regularity criteria for triangular and tetrahedral finite element partitions, Comput. Math. Appl. 55 (2008), 2227-2233. Zbl 1142.65443 
[3] Brandts, J., Korotov, S., Kř́ížEK, M., On the equivalence of ball conditions for simplicial finite elements in $\mathbf{R}^{d}$, Appl. Math. Lett. 22 (2009), 1210-1212. Zbl 1173.52301

[4] Brandts, J., Kř́́žEK, M., Gradient superconvergence on uniform simplicial partitions of polytopes, IMA J. Numer. Anal. 23 (2003), 489-505. Zbl 1042.65081

[5] Ciarlet, P. G., The Finite Element Method for Elliptic Problems, North-Holland, Amsterdam, 1978. Zbl 0383.65058

[6] Eriksson, F., The law of sines for tetrahedra and $n$-simplices, Geom. Dedicata 7 (1978), 71-80. Zbl 0375.50008

[7] Hannukainen, A., Korotov, S., KřížEK, M., On global and local mesh refinements by a generalized conforming bisection algorithm, J. Comput. Appl. Math. 235 (2010), 419-436.

[8] Lin, J., Lin, Q., Global superconvergence of the mixed finite element methods for 2-d Maxwell equations, J. Comput. Math. 21 (2003), 637-646. Zbl 1032.65101

[9] Rektorys, K., Survey of Applicable Mathematics, vol. 1, Kluwer Academic Publishers, Dordrecht, 1994. Zbl 0805.00002

[10] Schewchuk, J. R., What is a good linear finite element? Interpolation, conditioning, anisotropy, and quality measures, Preprint Univ. of California at Berkeley, 2002, $1-66$.

[11] ŽEní̌̌EK, A., The convergence of the finite element method for boundary value problems of a system of elliptic equations (in Czech), Apl. Mat. 14 (1969), 355-377. Zbl 0188.22604

[12] Zlámal, M., On the finite element method, Numer. Math. 12 (1968), 394-409. Zbl 0176.16001 